\title{
Prognostic Value of Ultrasound Stratigraphy in Long-Term Weight Loss: Results from a Nutritional Counseling Program
}

\author{
Luca De Toni Gabriel Cosmin Petre Andrea Garolla Ilaria De Santis \\ Umberto Valente Carlo Foresta Maurizio De Rocco Ponce \\ Unit of Andrology and Reproductive Medicine, Department of Medicine, University of \\ Padova, Padova, Italy
}

\section{Keywords}

Lifestyle intervention - Calorie restriction · Body composition · Adherence · Testosterone

\begin{abstract}
Background: Weight control through lifestyle interventions represents a suitable strategy to avoid the metabolic, endocrine, and reproductive comorbidities associated with overweight and obesity. Reduced testosterone $(\mathrm{T})$ levels are a worsening factor in overweight males. However, prognostic parameters of long-term weight loss are not readily available. Here, we tested the prognostic value of early variations of anthropometric and hormonal parameters, with a focus on ultrasound stratigraphy (US) and the reduction in body mass index (BMI) associated with nutritional counseling/lifestyle interventions at 6-month follow-up. Methods: Ninety-five male subjects (BMI $25-34.9 \mathrm{~kg} / \mathrm{m}^{2}$ ) who had undergone nutritional/lifestyle interventions, were retrospectively analyzed for: body weight and composition; US evaluation at the triceps (TRC), abdominal (ABD), and thigh (THI) areas; and circulating levels of T, luteinizing hormone, and follicle-stimulating hormone. Sixty patients (63.2\%) completed the 6-month follow-up program. Results: At 6 months, a significant reduction in BMI ( $26.38 \pm 1.55$ vs. 31.5 \pm 5.0 basal, $p<0.001)$ and increase in T levels ( $18 \pm 5.4$ vs. $9.5 \pm 2.3 \mathrm{nmol} / \mathrm{L}$ basal, $p=0.04)$ were observed. Subjects in the highest quartile of the BMI reduction at 6 months ( $\triangle B M I 6 \mathrm{mo}$ ), compared to the lowest, showed a significant difference at the 2-month follow-up variation of BMI ( $p=0.025)$, and fat and muscle thickness at the TRC (both $p<0.001)$ and ABD $(p<$ 0.001 and $p=0.002$, respectively) areas. Variation of TRC muscle thickness at 2 months was the only independent predictor of $\triangle \mathrm{BMI} 6 \mathrm{mo}$ in the multiple stepwise regression analysis. Conclusions: BMI evaluation and US represent useful monitoring tools in the follow-up of nutritional/lifestyle interventions for overweight-to-mildly obese patients. The important effects on motivation and adherence to the intervention program are to be considered.
\end{abstract}


De Toni et al.: Ultrasound Stratigraphy in Weight Loss Monitoring

\section{Introduction}

Sex hormones are acknowledged as major drivers of body composition in adults [1], as depicted by epidemiological and interventional data that suggest a bidirectional relationship between steroid pattern and overweight/obesity in both sexes [2]. Particularly in males, serum testosterone (T) levels are frequently reduced in both obese and overweight subjects, in parallel with increased estrogen levels, when compared to men with normal weight [3]. In this regard, abnormalities in the hypothalamic hypophysis testicular axis (HTPA) are frequently associated with weight gain and become clinically important with the onset of obesity [4]. On the other hand, a reduction in $\mathrm{T}$ is accompanied by a poorer quality of life and a reduced life expectancy, which represents a cardiovascular risk factor by itself and is causally involved with other comorbidities such as subfertility and muscle wasting [5].

The clinical evaluation and management of overweight/obese patients can thus benefit from the accurate evaluation of both hormonal profile and body composition [6]. Accordingly, a multidisciplinary approach combining a hypocaloric diet, systematic physical activity, and appropriate lifestyle interventions appears strategic for achieving and maintaining a proficient weight loss [7]. In spite of these efforts, there is a rate of discontinuation of hypocaloric protocols for obese/cardiovascular patients of $\geq 30 \%$ [8]. The main reasons for this dropout are weight loss targets not being reached, in spite of major efforts, and the discomfort derived from lifestyle interventions and calorie restriction [9].

With the aim of addressing these critical issues, many hypocaloric dietary approaches have been developed to obtain a relatively fast weight loss such as the Dukan Diet, the Atkins Diet, the 3 Day Diet, and Alternate Day Fasting, to cite a few [10]. However, there are major concerns about real weight-loss efficacy in relation to coexisting cardiovascular and diabetes risk factors, nutritional adequacy, dietary compliance, and quality of life [10]. Specifically, there is a substantial lack of reliable markers that are sufficiently informative about the efficacy of the adopted protocols, regarding control of body weight and composition and possible prognostic reflection on the long-term adherence of patients [11]. In this regard, the analysis of body composition by means of ultrasound stratigraphy (US), i.e., the echographyassisted measurement of fat and muscle tissues layers at different body sites of diagnostic value, is gaining interest in longitudinal studies because of its accuracy and reproducibility coupled with it being minimally invasive [12].

In this study, we evaluated the effects of nutritional counseling and lifestyle interventions in a group of male subjects affected by overweight to moderate obesity, who underwent 6 months of monitoring of their hormonal patterns, anthropometric characterization, and body composition. The assessment of body composition relied on both screening bioimpedentiometry and US [13]. The evaluation of these instrumental data was assessed for their potential early prognostic value within a weight loss program.

\section{Materials and Methods}

\section{Patients}

This was a retrospective study conducted at the Foresta Foundation O.N.L.U.S. (Padova, Italy) from February 2014 to December 2018. The Foundation offers a pro bono nutritional counseling service for spontaneous attendance. The study involved a cohort of 95 Caucasian males living in the Veneto region, who were affected by body mass index (BMI) values indicating overweight $(25<\mathrm{BMI}<29.9)$ or class I obesity $(30<\mathrm{BMI}<34.9)[14]$ and presented at the Foundation with the aim of weight control. 
The exclusion criteria were: a history of cardiovascular, renal, or hepatic disease; type 1 or 2 diabetes mellitus, hormonal replacement therapy with testosterone; a diagnosis of Klinefelter syndrome; a previous history of eating disorders and alcohol abuse, or the use of recreational substances resulting in unstable body weight for 3 months before the start of the study ( $>4 \mathrm{~kg}$ weight loss or weight gain).

The nutritional counseling program consisted of an outpatient evaluation at study entry, at which pathological proximate and remote anamnesis, pathological familiarity, an evaluation of ongoing pharmacological therapies, an assessment for the risk of malnutrition, weight history, gastrointestinal symptomatology, allergies/intolerances, socioeconomic data, and the consumption of alcohol/recreational substances were recorded. Nutritional history was also taken into consideration, energy and nutrient intake, general eating habits and, specifically, in cases of possible underestimation of nutritional intake, keeping a 7-days food diary was prescribed [15]. Anthropometric measurements were carried out according to the International Society for the Advancement of Kinanthropometry (ISAK) [16] and included the evaluation of weight, height, BMI, and waist circumference. The evaluation of body composition included both indirect data from screening bioelectrical impedance body composition monitoring (BIBCM; Kardascan BF511, Omron, Lacchiarella, Italy) and direct data obtained from US (see below). Each subject was given a personalized low-calorie $(-30 \%$ of the estimated total daily energy expenditure)/moderately high-protein $(+15-18 \%$ protein energy intake) diet plan that was divided into 3 main meals and 2 snacks [17]. In addition, systematic mixed endurance and strength physical activity was recommended 3 times a week for at least 45 min per session [18].

Each subject was followed for a total period of 6 months, with interviews scheduled every 2 months. The longitudinal change of body composition and anthropometric and humoral parameters were evaluated at each interview (i.e., at baseline and then at 2, 4 and 6 months).

\section{Ultrasound Stratigraphy}

Evaluation of body composition by US was performed with the patient in supine position. All Doppler ultrasounds were carried out by the same experienced operator (L.D.T., G.C.P., U.V.), with a high-resolution echo color Doppler device (iU22 Philips, Eindhoven, The Netherlands or Aplio XV Toshiba, Tokyo, Japan) equipped with a probe (7-13 MHz; axial resolution $<0.1 \mathrm{~mm}$ ). Measurements were done by gently applying the transducer onto the skin that was coated by a thin layer of water-soluble gel to avoid compression of the subcutaneous fat. The transducer was held orthogonal to the skin surface to ensure precise depth analyses and avoid transmission parallax error. During the measurement, the transducer was glided slightly back and forth along the skin surface $( \pm 10 \mathrm{~mm})$ to provide local averaging of the measurements over a period of 3-5 s. The measurement was performed at 3 diagnostic sites: triceps (TRC), abdominal (ABD), and thigh (THI) areas. The thickness of the subcutaneous adipose and muscle layer (from the lower end of the fat layer to the long bone surface) was recorded for TRC and THI (Fig. 1A, B). For ABD area, the thickness of the subcutaneous adipose, rectus abdominis muscle, and omental adipose tissue (OAT) layers (from the lower end of the rectus abdominis to the body of the vertebra surface) was recorded (Fig. 1C).

\section{Hormone Assays}

Blood was collected in the fasting state between 08.00 and 10.00 a.m. Serum folliclestimulating hormone (FSH), luteinizing hormone ( $\mathrm{LH})$, and total $\mathrm{T}$ were evaluated by commercial electrochemiluminescence immunoassay methods (Elecsys 2010, Roche Diagnostics, Mannheim, Germany). For all parameters, the intra- and interassay coefficients of variation were $<8$ and $10 \%$, respectively. All determinations were performed according to the manufacturer's instructions. 
A
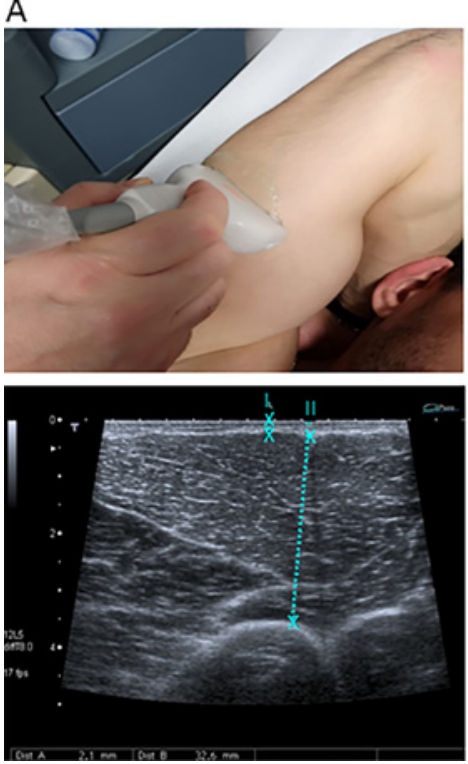

B
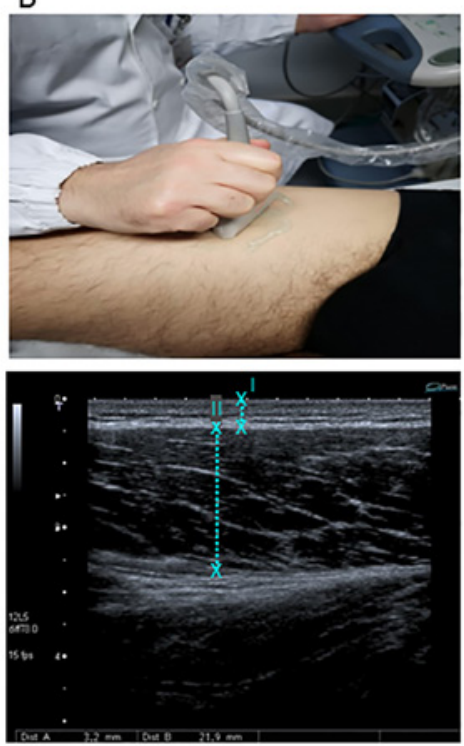

C
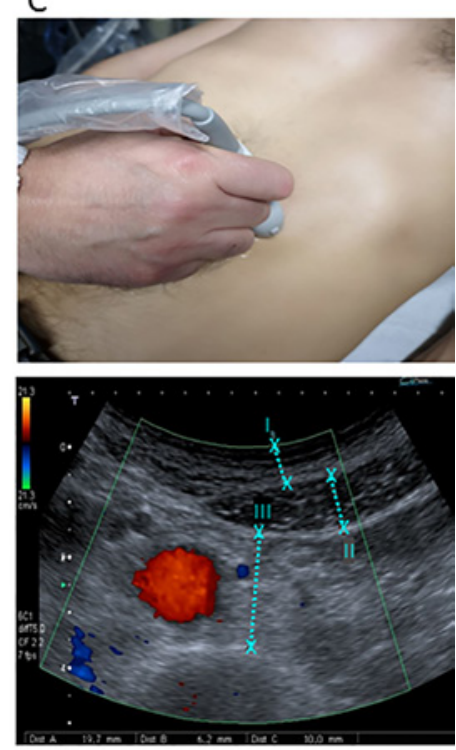

Fig. 1. Triceps (A), thigh (B) and abdominal (C) area measurements for ultrasound stratigraphy. The lower panels show examples of evaluation of the thickness of the subcutaneous adipose (distance I), muscle (distance II), and omental adipose (distance III) layers.

\section{Statistical Analysis}

Pearson's $\chi^{2}$ test, or Fisher's exact test when the expected frequency was $\leq 5$, was used to examine the differences in the distribution of frequencies. The differences in anthropometric and hormonal parameters between $>2$ groups were evaluated by analysis of variance using the Bonferroni-Holm correction. The associations between early variations of anthropometric parameters and long-term BMI reduction were estimated by computing the odds ratios (ORs) and their 95\% confidence intervals (CIs) in logistic regression analyses. $p$ values $<0.05$ were considered statistically significant. Statistical analyses were performed using SPSS v20.0 for Windows (SPSS, Chicago, IL, USA).

\section{Results}

Sixty out of 95 male patients enrolled in the study (mean age $46.0 \pm 15.4$ years; BMI at recruitment $31.5 \pm 5.0$ ) completed the 6-month follow-up, with an overall dropout rate of $36.8 \%$, comparable to other similar studies involving the association between caloric restriction and training protocol [8]. The anthropometric and hormonal parameters of the study population at both baseline and at the end of the observation period are reported in Table 1. Nutritional/lifestyle interventions obtained an average reduction of body weight of $6.8 \pm 7.1 \mathrm{~kg}$ at 6 months. This resulted in a significant reduction in BMI, waist circumference, and fat mass percentage, and a corresponding increase of lean mass percentage at screening BIBCM compared to baseline values. A reduction in the percentage of OAT was also observed, but not at a significant level.

Results of US evaluation showed a significant reduction in subcutaneous fat thickness and a corresponding increase in muscle thickness at both the TRC and ABD areas, compared to baseline. THI area data showed a similar trend, without achieving significant differences. 
De Toni et al.: Ultrasound Stratigraphy in Weight Loss Monitoring

Table 1. Anthropometric and hormonal characteristics of 60 overweight male patients accessing nutritional counseling, at baseline and after 6 months of follow-up

\begin{tabular}{lccc}
\hline & Baseline & $\begin{array}{c}\text { At 6-month } \\
\text { follow-up }\end{array}$ & $p$ value \\
& & & \\
\hline Age, years & $46.0 \pm 15.4$ & - & - \\
Height, m & $1.76 \pm 0.05$ & - & - \\
Weight, kg & $97.1 \pm 17.1$ & $94.38 \pm 13.23$ & $\mathbf{0 . 0 0 5}$ \\
BMI, kg/m ${ }^{2}$ & $31.5 \pm 5.0$ & $26.38 \pm 1.55$ & $<\mathbf{0 . 0 0 1}$ \\
Waist circumference, cm & $112.7 \pm 12.4$ & $102.43 \pm 6.90$ & $\mathbf{0 . 0 1 2}$ \\
Lean mass, \% & $31.1 \pm 3.7$ & $33.76 \pm 2.38$ & $\mathbf{0 . 0 4 4}$ \\
Fat mass, \% & $32.8 \pm 6.5$ & $27.04 \pm 5.39$ & $\mathbf{0 . 0 3 7}$ \\
OAT mass, \% & $15.4 \pm 4.9$ & $11.57 \pm 3.82$ & 0.051 \\
TRC Fat, mm & $6.8 \pm 2.0$ & $5.30 \pm 0.98$ & $\mathbf{0 . 0 3 1}$ \\
TRC Lean, mm & $30.3 \pm 6.0$ & $33.85 \pm 1.33$ & $\mathbf{0 . 0 4 5}$ \\
ABD Fat, mm & $31.6 \pm 6.6$ & $23.60 \pm 2.62$ & $\mathbf{0 . 0 0 1}$ \\
ABD Lean, mm & $11.6 \pm 2.9$ & $14.48 \pm 2.07$ & $\mathbf{0 . 0 1 2}$ \\
ABD OAT, mm & $117.9 \pm 21.9$ & $103.01 \pm 9.43$ & $\mathbf{0 . 0 3 8}$ \\
THI Fat, mm & $13.9 \pm 3.5$ & $12.73 \pm 4.26$ & 0.579 \\
THI Lean, mm & $43.8 \pm 10.9$ & $50.29 \pm 4.98$ & 0.071 \\
Testosterone, mmol/L & $9.5 \pm 2.3$ & $18 \pm 5.4$ & $\mathbf{0 . 0 4 0}$ \\
LH, IU/L & $3.5 \pm 1.2$ & $2.5 \pm 0.8$ & $\mathbf{0 . 0 5 0}$ \\
FSH, IU/L & $5.3 \pm 2.6$ & $6.0 \pm 3.4$ & 0.090 \\
\hline
\end{tabular}

Significant differences between 6-month follow-up and baseline data appear in bold type. OAT, omental adipose tissue; TRC Fat, subcutaneous fat thickness at triceps area; TRC Lean, muscle thickness at triceps area; ABD Fat, subcutaneous fat thickness of abdominal area; ABD Lean, rectus abdominis muscle thickness; ABD OAT, omental fat thickness; THI Fat, subcutaneous fat thickness at upper third of the thigh area; THI Lean, muscle thickness at upper third of the thigh area; $\mathrm{LH}$, luteinizing hormone; FSH, follicle-stimulating hormone.

Hormonal parameters showed that $35 / 60$ patients had serum $\mathrm{T}$ levels below the normal threshold of $10.4 \mathrm{nmol} / \mathrm{L}$ (mean $9.5 \pm 2.3 \mathrm{nmol} / \mathrm{L}$ ). Adherence to 6 months of nutritional counseling was associated with a significant increase in serum $\mathrm{T}$ and a slight but significant decrease in LH compared to baseline. A marginally significant decrease in FSH was observed.

A time course analysis of anthropometric and hormonal parameters was performed, showing significant variation during the follow-up period (Fig. 2). Taking the study group as a whole, each parameter achieved a significant difference from the baseline level only after 6 months from the beginning of the nutritional and lifestyle interventions, with a nonapparent variation at earlier times. Based on this, to identify possible parameters with early prognostic value for long-term reduction in body weight, the following approach was adopted. The 2 -month variations in BMI $(\Delta \mathrm{BMI} 2 \mathrm{mo})$, waist circumference $(\Delta$ Waist $2 \mathrm{mo})$, fat mass percentage ( $\Delta$ Fat $2 \mathrm{mo}$ ), lean mass percentage ( $\Delta$ Lean $2 \mathrm{mo}$ ), serum $\mathrm{T}(\Delta \mathrm{T} 2 \mathrm{mo})$, and fat and muscle thickness at the TRC ( $\triangle$ TRC Fat 2 mo and $\triangle$ TRC Lean 2 mo, respectively) and ADB $(\triangle \mathrm{ABD}$ Fat 2 mo and $\triangle \mathrm{ADB}$ Lean $2 \mathrm{mo}$, respectively) areas as well as OAT thickness ( $\triangle \mathrm{ADB}$ OAT $2 \mathrm{mo}$ ), were stratified according to quartiles (Q) of the 6-month variations in BMI $(\triangle \mathrm{BMI}$ 6 mo). Subsequently, for each parameter, the mean value derived from $\Delta B M I 6$ mo quartile subdivisions was compared to that of $\mathrm{Q} 4$ as the reference (corresponding to the lowest/no reduction of BMI). Results are reported in Table 2. Significant differences, compared to Q4 $\Delta$ BMI 6 mo, were observed for $\Delta$ BMI 2 mo at Q1 $(p=0.025), \Delta$ TRC Fat 2 mo at Q1 and Q2 $(p<0.001$ and $p=0.020$, respectively), $\Delta$ TRC Lean 2 mo at Q1 and Q2 ( $p<0.001$ for both), $\triangle \mathrm{ABD}$ Fat 2 mo for Q1 ( $p<0.001)$, and $\triangle \mathrm{ADB}$ Lean 2 mo for Q1 and Q2 ( $p=0.002$ and $p=0.004$, respectively). Importantly, all $\triangle$ BMI 6 mo did not differ for possible confounders such as the 


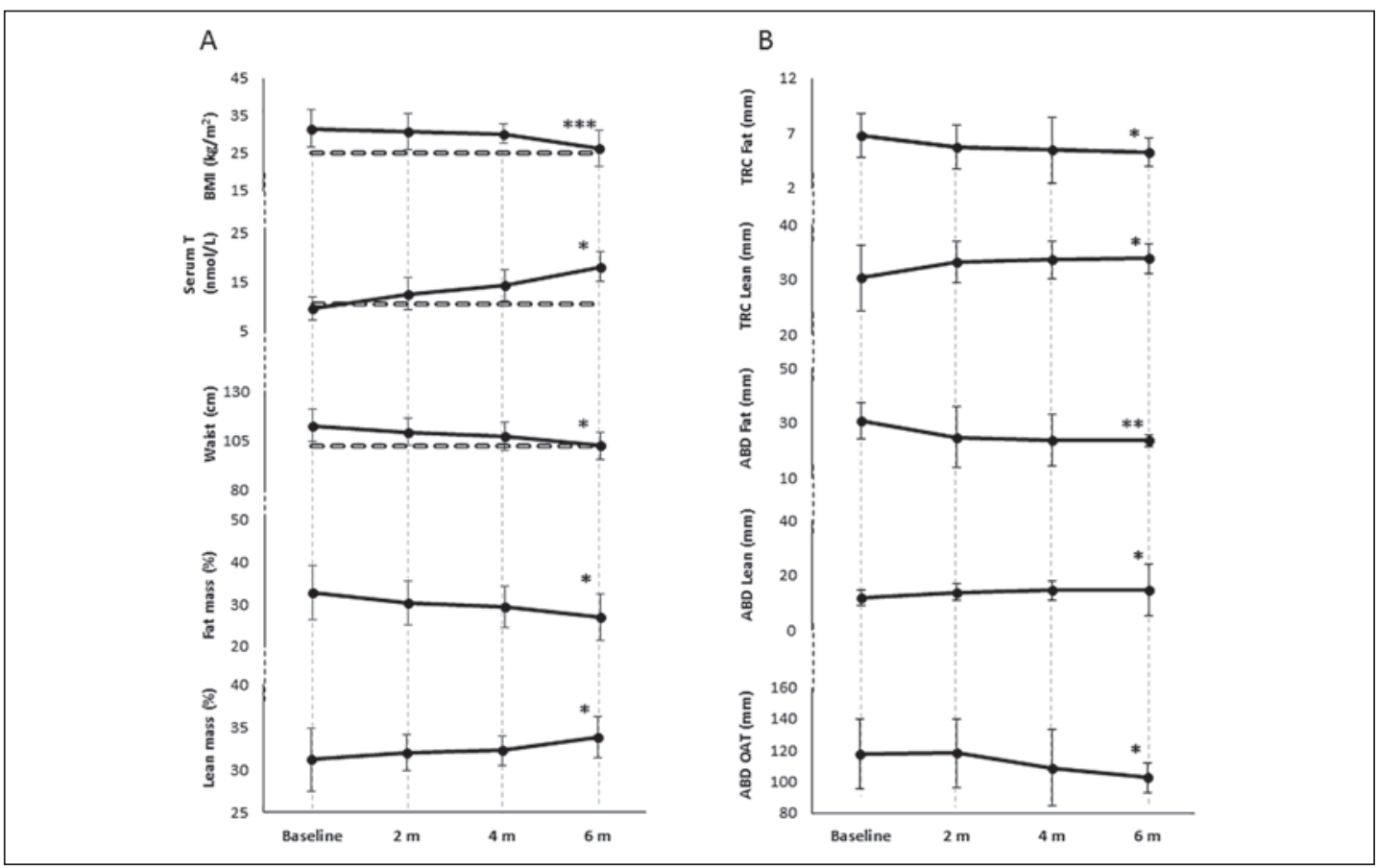

Fig. 2. Time course of body mass index (BMI), serum testosterone (T), waist circumference (waist), percentage of fat mass, and percentage of fat mass lean mass $(\mathbf{A})$, and subcutaneous fat, and muscle thickness at the triceps area (TRC Fat and TRC Lean, respectively) and subcutaneous fat, rectus abdominis, and omental adipose tissue thicknesses at the abdomen area (ABD Fat, ABD Lean, and ABD OAT, respectively) (B). All parameters were evaluated at recruitment (Baseline) and at 2, 4, and 6 months ( $2 \mathrm{~m}, 4 \mathrm{~m}$, and $6 \mathrm{~m}$, respectively) of follow-up. ${ }^{*} p<0.05$; $^{* *} p<0.01$; ${ }^{* * *} p<0.001$ versus baseline.

age of the patient at recruitment, the baseline BMI, and the prevalence of hypogonadism (all $p>0.05$ ).

In addition, in order to define a possible prognostic value from these results, each the of the 2-month variations of the aforementioned parameters was stratified into distribution quartiles and the prevalence of patients belonging to Q1 $\triangle \mathrm{BMI} 6$ mo (corresponding to the highest observed reduction in BMI at 6 months) was calculated with the related OR. Results are reported in Table 3. Significant differences in clustering of Q1 $\triangle B M I 6$ mo patients and OR were observed for $\triangle$ BMI 2 mo, $\triangle$ TRC Fat 2 mo, $\triangle$ TRC Lean 2 mo, $\triangle$ ABD Fat 2 mo, and $\triangle$ ADB Lean 2 mo from Q1 versus Q4. Interestingly, the differential clustering for $\triangle \mathrm{TRC}$ Fat 2 mo, $\triangle T R C$ Lean 2 mo, $\triangle A B D$ Fat 2 mo, and $\triangle A D B$ Lean 2 mo remained significant even after the Q1 + $\mathrm{Q} 2$ versus $\mathrm{Q} 3+\mathrm{Q} 4$ comparison, corresponding to a less stringent condition.

Finally, multiple stepwise regression analysis, including age, baseline BMI, and serum $\mathrm{T}$ as possible confounders, showed that $\triangle \mathrm{TRC}$ Lean 2 mo was the only independent predictor of $\Delta$ BMI 6 mo $(\beta=-0.611 ; t=-4.307 ; p<0.001)$.

\section{Discussion}

In this study, we provide evidence that a 6-month nutritional counseling program, combined with lifestyle interventions, is efficient in improving weight loss and androgen levels when applied to overweight/mildly obese patients. Furthermore, we show that an early reduction in BMI and US are significant predictors of long-term BMI reduction. 


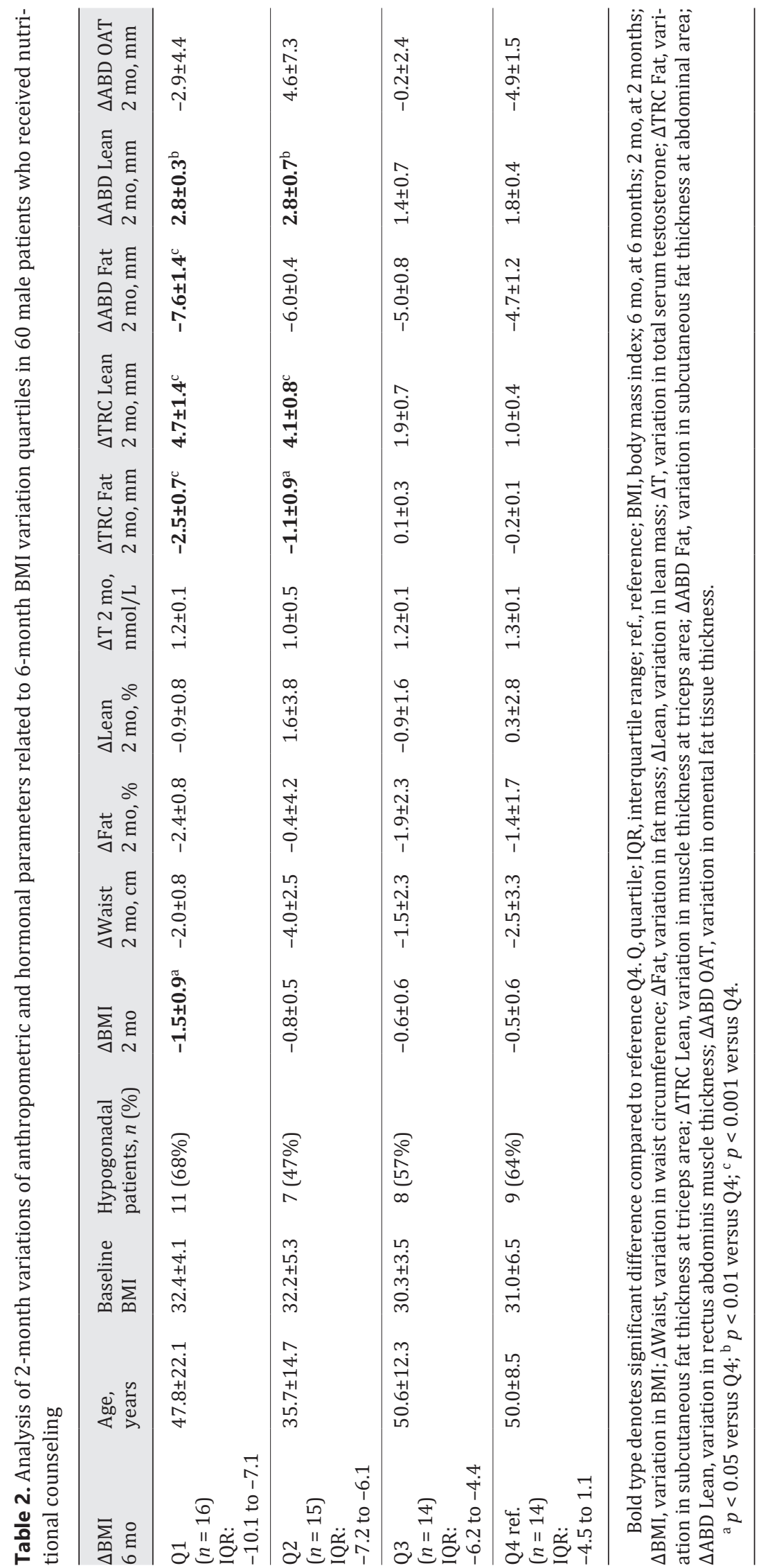


Table 3. Prognostic value of 2-month variations in anthropometric parameters for the top quartile of the reduction in BMI at 6 months

\begin{tabular}{|c|c|c|c|}
\hline $\begin{array}{l}\text { Parameter } \\
Q \text { (interquartile range) }\end{array}$ & $\begin{array}{l}\text { Comparison of quartiles } \\
(n / N) \text { in } \mathrm{Q} 1 \Delta \mathrm{BMI} 6 \mathrm{mo}\end{array}$ & OR $(95 \% \mathrm{CI})$ & $p$ value \\
\hline \multicolumn{4}{|l|}{$\triangle B M I 2 \mathrm{mo}$} \\
\hline Q2 (-1.4 to -0.8$)$ & Q1 (7/15) vs. Q4 (2/15) & $5.69(0.94-34.46)$ & 0.0464 \\
\hline \multicolumn{4}{|l|}{$\mathrm{Q} 3(-0.7$ to -0.2$)$} \\
\hline $\mathrm{Q} 4(-0.1$ to -0.0$)$ & $\mathrm{Q} 1+2(10 / 30)$ vs. $Q 3+4(6 / 30)$ & $3.00(0.68-13.31)$ & 0.1432 \\
\hline \multicolumn{4}{|l|}{$\Delta$ Waist $2 \mathrm{mo}, \mathrm{cm}$} \\
\hline Q2 $(-3.2$ to -2.0$)$ & Q1 (1/17) vs. Q4 (1/11) & $0.63(0.04-11.16)$ & 0.7475 \\
\hline \multicolumn{4}{|l|}{ Q3 (-1.9 to -1.0$)$} \\
\hline Q4 (-0.9 to 2.0$)$ & $\mathrm{Q} 1+2(11 / 32)$ vs. $\mathrm{Q} 3+4(5 / 28)$ & $2.41(0.72-8.09)$ & 0.1489 \\
\hline \multicolumn{4}{|l|}{$\begin{array}{l}\Delta \text { Fat } 2 \mathrm{mo}, \% \\
\mathrm{Q} 1(-5.8 \text { to }-2.8)\end{array}$} \\
\hline $\mathrm{Q} 2(-2.7$ to -2.2$)$ & Q1 (7/17) vs. Q4 (2/15) & $4.55(0.77-26.84)$ & 0.0804 \\
\hline \multicolumn{4}{|l|}{ Q3 $(-2.1$ to -1.1$)$} \\
\hline $\mathrm{Q} 4(-1.1$ to 4.4$)$ & $\mathrm{Q} 1+2(9 / 30)$ vs. $\mathrm{Q} 3+4(6 / 30)$ & $1.71(0.52-5.62)$ & 0.3711 \\
\hline \multicolumn{4}{|l|}{$\begin{array}{l}\Delta \text { Lean } 2 \text { mo, } \% \\
\text { Q1 }(-3.2 \text { to }-1.2)\end{array}$} \\
\hline Q2 (-1.1 to -0.4$)$ & Q1 (2/15) vs. Q4 (4/15) & $0.42(0.06-2.77)$ & 0.3613 \\
\hline \multicolumn{4}{|l|}{ Q3 (-0.3 to -0.9$)$} \\
\hline $\mathrm{Q} 4(-0.8$ to 8.0$)$ & $\mathrm{Q} 1+2(7 / 30)$ vs. $\mathrm{Q} 3+4(9 / 30)$ & $1.41(0.45-4.45)$ & 0.5593 \\
\hline \multicolumn{4}{|l|}{$\begin{array}{l}\Delta T 2 \mathrm{mo}, \mathrm{nmol} / \mathrm{L} \\
\mathrm{Q} 1(0.1 \text { to } 1.2)\end{array}$} \\
\hline Q2 (1.2 to 1.3$)$ & Q1 (5/15) vs. Q4 (3/17) & $2.33(0.45-12.09)$ & 0.3065 \\
\hline \multicolumn{4}{|l|}{ Q3 (1.3 to 1.4$)$} \\
\hline Q4 (1.4 to 1.5$)$ & Q1+2 (9/28) vs. Q3+4 (7/32) & $1.69(0.53-5.36)$ & 0.3696 \\
\hline \multicolumn{4}{|l|}{$\begin{array}{l}\triangle T R I C \text { Lean } 2 \mathrm{mo}, \mathrm{mm} \\
\mathrm{Q} 1(0.3 \text { to } 1.4)\end{array}$} \\
\hline Q2 (1.5 to 2.9) & Q1 (1/17) vs. Q4 (10/13) & $53.33(4.85-586.24)$ & $<0.001$ \\
\hline \multicolumn{4}{|l|}{ Q3 (3.0 to 4.2 ) } \\
\hline $\mathrm{Q} 4$ (4.3 to 6.5$)$ & $\mathrm{Q} 1+2(2 / 30)$ vs. $\mathrm{Q} 3+4(14 / 30)$ & $7.88(1.55-40.09)$ & 0.0065 \\
\hline \multicolumn{4}{|l|}{$\begin{array}{l}\triangle T R I C \text { Fat } 2 \mathrm{mo}, \mathrm{mm} \\
\mathrm{Q} 1(-3.8 \text { to }-1.9)\end{array}$} \\
\hline Q2 (-1.8 to -0.5$)$ & Q1 (9/15) vs. Q4 (13/15) & $21.00(2.16-204.62)$ & 0.0019 \\
\hline \multicolumn{4}{|l|}{ Q3 $(-0.4$ to -0.2$)$} \\
\hline $\mathrm{Q} 4(-0.1$ to 0.5$)$ & $\mathrm{Q} 1+2(13 / 30)$ vs. $\mathrm{Q} 3+4(3 / 30)$ & $6.88(1.71-27.75)$ & 0.0035 \\
\hline \multicolumn{4}{|l|}{$\begin{array}{l}\triangle A B D \text { Fat } 2 \mathrm{mo}, \mathrm{mm} \\
\mathrm{Q} 1(-9.6 \text { to }-6.9)\end{array}$} \\
\hline Q2 (-6.8 to -5.9$)$ & Q1 (11/17) vs. Q4 (1/15) & $25.67(2.68-245.85)$ & $<0.001$ \\
\hline \multicolumn{4}{|l|}{ Q3 (-5.8 to -4.7$)$} \\
\hline Q4 (-4.7 to -3.6$)$ & $\mathrm{Q} 1+2(14 / 30)$ vs. $\mathrm{Q} 3+4(2 / 30)$ & $12.25(2.46-60.91)$ & 0.001 \\
\hline \multicolumn{4}{|l|}{$\begin{array}{l}\triangle A B D \text { Lean } 2 \mathrm{mo}, \mathrm{mm} \\
\mathrm{Q} 1(0.4 \text { to } 1.5)\end{array}$} \\
\hline Q2 (1.6 to 2.3 ) & Q1 (1/17) vs. Q4 (8/15) & $8.29(1.91-175.35)$ & 0.0029 \\
\hline \multicolumn{4}{|l|}{ Q3 (2.4 to 2.8 ) } \\
\hline Q4 (2.9 to 3.9 ) & $\mathrm{Q} 1+2(3 / 32)$ vs. $\mathrm{Q} 3+4(13 / 28)$ & $8.38(2.06-34.03)$ & 0.0012 \\
\hline
\end{tabular}


De Toni et al.: Ultrasound Stratigraphy in Weight Loss Monitoring

Table 3 (continued)

\begin{tabular}{|c|c|c|c|}
\hline $\begin{array}{l}\text { Parameter } \\
\text { Q (interquartile range) }\end{array}$ & $\begin{array}{l}\text { Comparison of quartiles } \\
(n / N) \text { in } \mathrm{Q} 1 \Delta \mathrm{BMI} 6 \mathrm{mo}\end{array}$ & OR $(95 \% \mathrm{CI})$ & $p$ value \\
\hline \multicolumn{4}{|l|}{$\begin{array}{l}\triangle A B D \text { OAT } 2 \mathrm{mo}, \mathrm{mm} \\
\mathrm{Q} 1(-10.0 \text { to }-3.3)\end{array}$} \\
\hline $\begin{array}{l}\text { Q2 }(-3.2 \text { to }-2.2) \\
\text { Q3 }(-2.1 \text { to }-0.7)\end{array}$ & Q1 (6/15) vs. Q4 (3/15) & $2.67(0.52-13.66)$ & 0.2320 \\
\hline Q4 (0.6 to 16.5$)$ & $\mathrm{Q} 1+2(10 / 30)$ vs. $\mathrm{Q} 3+4(6 / 30)$ & $2.00(0.62-6.47)$ & 0.2429 \\
\hline
\end{tabular}

Bold type denotes significant difference in patient's prevalence among indicated quartiles. BMI, body mass index; Q, quartile; 6 mo, at 6 months; 2 mo, at 2 months; $\Delta \mathrm{BMI}$, variation in BMI; $\Delta$ Waist, variation in waist circumference; $\Delta$ Fat, variations in fat mass; $\Delta$ Lean, variation in lean mass; $\Delta \mathrm{T}$, variation in total serum testosterone; $\triangle \mathrm{TRC}$ Fat, variation in subcutaneous fat thickness at triceps area; $\triangle \mathrm{TRC}$ Lean, variation in muscle thickness at triceps area; $\triangle \mathrm{ABD}$ Fat, variation in subcutaneous fat thickness at abdominal area; $\triangle \mathrm{ABD}$ Lean, variation in rectus abdominis muscle thickness; $\triangle \mathrm{ABD} O \mathrm{OAT}$, variation in omental fat tissue thickness.

The positive correlation between adherence to a dietary/lifestyle intervention program and weight loss outcomes has been acknowledged by several studies [19-21]. Accordingly, on the one hand, the cost-effectiveness of the intervention may be improved by reducing discontinuation [22]. On the other hand, the availability of predictive factors for weight loss efficacy provides motivational elements to improve adherence to intervention and/or identify switching strategies to sustain the benefit of the intervention [23, 24]. In particular, the extent of the initial treatment response, e.g., early weight loss, has been associated with the low discontinuation rate and greater efficacy of long-term weight loss interventions [25-27]. Fabricatore et al. [28] performed a retrospective analysis of this trial on pharmacotherapy and behavior therapy for weight loss for the treatment of severely obese patients $(30 \leq \mathrm{BMI} \leq$ 45). They reported that each additional day of food recording during the first 3 weeks of treatment was associated with a $7 \%$ increase in the odds of achieving $\geq 5 \%$ weight loss at 1 year. Furthermore, in the whole group, weight loss at 3 weeks was the most significant predictor of weight loss achievement at 1 year (OR 1.73) [28]. We observed parallel results, since patients clustered in the top quartile of BMI reduction achieved within 2 months (compared to the early mild/no reduction of the bottom quartile) showed a nearly 6-fold probability of obtaining the greatest BMI reduction at 6 months. Importantly, the possible confounding effect of age, baseline BMI, and serum T levels below the normal range, was ruled out by comparing these parameters after unbiased clustering of patients according to $\Delta \mathrm{BMI}$ 2 mo. Major discrepancies with the results observed in the above studies possibly relate to differences in study characteristics; their patients were severely obese at baseline and a mixed pharmacological/behavior treatment approach was implemented [27, 28]. Nevertheless, the significant correlation reported in our study was lost when milder reductions in $\mathrm{BMI}$ at 2 months were considered, e.g., comparing the top 2 with the bottom $2 \Delta \mathrm{BMI} 2 \mathrm{mo}$ values. Indeed, this evidence likely relies on raw BMI data being a poor representation of the locoregional distribution of body mass and its composition [29].

More informative data were apparently provided by anthropometric variations evaluated by US. This is an ultrasound imaging-based evaluation technique applied for tissue thickness measurements at a set of standardized sites [30]. Originally applied in groups of athletes and normal-weight persons, and successively in overweight or obese subjects, US has gained attention for its relatively high precision and accuracy, and now software-aided automated instruments have been launched on the market for possible use in the lab and in the field [30-32]. By applying US evaluation criteria to the follow-up of patients, we observed a 
strict correlation between early variations in subcutaneous fat and muscle at the TRC and $\mathrm{ABD}$ areas and 6-month variations in BMI. Intriguingly, multiple stepwise regression analysis revealed that the 2-month variation in TRC muscle thickness was the only independent predictor of BMI variation at the 6-month follow-up. In parametric terms, 2-month increases in TRC muscle $>1.5 \mathrm{~mm}$ (compared to lesser increases) were associated with a nearly 8 -foldhigher probability of achieving a reduction in BMI of $>7$ units at 6 months. This evidence requires further confirmation, but it represents a novel cue for 2 reasons. First, it represents an early parameter that addresses the efficacy of nutritional/lifestyle interventions. Second, the continuous monitoring of and feedback from patients may represent a useful tool to improve their involvement and motivation, and, ultimately, support them in adhering to the intervention program.

We acknowledge the study limitations. There was a small sample size that may have led to overestimation in the results. More studies on larger cohorts are warranted to better address the specificity and accuracy of these preliminary observations. In addition, longer follow-up observation should be scheduled to verify the stability of the anthropometric improvements observed at 6 months.

In conclusion, we showed that frequent anthropometric monitoring by means of classical BMI evaluation and US represent useful tools for the follow-up of nutritional/lifestyle counseling applied to overweight/mildly obese patients. The important effects on motivation and adherence to the interventions program are to be considered. Further studies on larger study cohorts are required to validate the efficacy and consistency of these preliminary data.

\section{Acknowledgements}

We thank Dr. Alessandro Bertoldo and Dr. Nicola Caretta for the helpful discussion.

\section{Statement of Ethics}

Retrospective access to clinical data of patients was allowed after sign of an informed consent. The study was part of an andrological survey program, approved by the local Ethics Committee (Protocol number \#2208 and successive amendments).

\section{Disclosure Statement}

The authors have nothing to disclose. None of the authors had potential conflicts of interest due to relationships with commercial/corporate interests. No financial assistance was received in support of the study.

\section{Author Contributions}

L. De Toni and G.C. Petre contributed equally to the conception and design of the research; A. Garolla contributed to the design of the research; U. Valente and I. De Santis contributed to the acquisition and analysis of the data; C. Foresta and M. De Rocco Ponce contributed to the interpretation of the data; and L. De Toni and G.C. Petre drafted the manuscript. All authors critically revised the manuscript, agree to be fully accountable for ensuring the integrity and accuracy of the work, and read and approved the final manuscript. 
De Toni et al.: Ultrasound Stratigraphy in Weight Loss Monitoring

\section{References}

1 Lee K, Sung J, Song YM. Genetic and environmental influence on the association between testosterone, sex hormone-binding globulin and body composition in Korean men. Clin Endocrinol (Oxf). 2015 Aug;83(2):236-45.

2 Escobar-Morreale HF. The Role of Androgen Excess in Metabolic Dysfunction in Women: Androgen Excess and Female Metabolic Dysfunction. Adv Exp Med Biol. 2017;1043:597-608.

3 Kelly DM, Jones TH. Testosterone and obesity. Obes Rev. 2015 Jul;16(7):581-606.

4 Nieschlag E. Current topics in testosterone replacement of hypogonadal men. Best Pract Res Clin Endocrinol Metab. 2015 Jan;29(1):77-90.

5 Ebrahimi F, Christ-Crain M. Metabolic syndrome and hypogonadism - two peas in a pod. Swiss Med Wkly. 2016 Mar; 146:w14283.

6 Hackett G, Cole N, Bhartia M, Kennedy D, Raju J, Wilkinson P. Testosterone replacement therapy with longacting testosterone undecanoate improves sexual function and quality-of-life parameters vs. placebo in a population of men with type 2 diabetes. J Sex Med. 2013 Jun;10(6):1612-27.

7 Kushner RF, Ryan DH. Assessment and lifestyle management of patients with obesity: clinical recommendations from systematic reviews. JAMA. 2014 Sep;312(9):943-52.

8 Zapico AG, Benito PJ, González-Gross M, Peinado AB, Morencos E, Romero B, et al. Nutrition and physical activity programs for obesity treatment (PRONAF study): methodological approach of the project. BMC Public Health. 2012 Dec;12(1):1100.

9 Solianik R, Sujeta A, Čekanauskaitè A. Effects of 2-day calorie restriction on cardiovascular autonomic response, mood, and cognitive and motor functions in obese young adult women. Exp Brain Res. 2018 Aug; 236(8):2299-308.

10 Astrup A, Meinert Larsen T, Harper A. Atkins and other low-carbohydrate diets: hoax or an effective tool for weight loss? Lancet. 2004 Sep;364(9437):897-9.

11 Andreoli A, Garaci F, Cafarelli FP, Guglielmi G. Body composition in clinical practice. Eur J Radiol. 2016 Aug; 85(8):1461-8.

12 Störchle P, Müller W, Sengeis M, Ahammer H, Fürhapter-Rieger A, Bachl N, et al. Standardized Ultrasound Measurement of Subcutaneous Fat Patterning: High Reliability and Accuracy in Groups Ranging from Lean to Obese. Ultrasound Med Biol. 2017 Feb;43(2):427-38.

13 Buffa R, Saragat B, Cabras S, Rinaldi AC, Marini E. Accuracy of specific BIVA for the assessment of body composition in the United States population. PLoS One. 2013;8(3):e58533.

14 World Health Organization (WHO). Technical Report Series 894: Obesity: Preventing and Managing the Global Epidemic. Geneva: World Health Organization; 2000.

15 Kothandan SK. School-based interventions versus family-based interventions in the treatment of childhood obesity - a systematic review. Arch Public Health. 2014 Jan;72(1):3.

16 Marfell-Jones M, Stewart FG, Ridder AD, editors. International Standards for Anthropometric Assessment. International Society for the Advancement of Kinanthropometry; 2011.

17 Dewar DL, Morgan PJ, Plotnikoff RC, Okely AD, Collins CE, Batterham M, et al. The Nutrition and Enjoyable Activity for Teen Girls Study: a cluster randomized controlled trial. Am J Prev Med. 2013 Sep;45(3):313-7.

18 Spear BA, Barlow SE, Ervin C, Ludwig DS, Saelens BE, Schetzina KE, et al. Recommendations for treatment of child and adolescent overweight and obesity. Pediatrics. 2007 Dec;120 Suppl 4:S254-88.

19 Fontaine KR, Cheskin LJ. Self-efficacy, attendance, and weight loss in obesity treatment. Addict Behav. 1997 Jul-Aug;22(4):567-70.

20 Honas JJ, Early JL, Frederickson DD, O’Brien MS. Predictors of attrition in a large clinic-based weight-loss program. Obes Res. 2003 Jul;11(7):888-94.

21 Carels RA, Cacciapaglia HM, Douglass OM, Rydin S, O’Brien WH. The early identification of poor treatment outcome in a women's weight loss program. Eat Behav. 2003 Sep;4(3):265-82.

22 Bennett GA, Jones SE. Dropping out of treatment for obesity. J Psychosom Res. 1986;30(5):567-73.

23 LaPorte DJ, Stunkard AJ. Predicting attrition and adherence to a very-low-calorie diet: a prospective investigation of the eating inventory. Int J Obes. 1990 Mar;14(3):197-206.

24 Pekarik G, Blodgett C, Evans RG, Wierzbicki M. Variables related to continuance in a behavioral weight loss program. Addict Behav. 1984;9(4):413-6.

25 Greenberg I, Stampfer MJ, Schwarzfuchs D, Shai I; DIRECT Group. Adherence and success in long-term weight loss diets: the dietary intervention randomized controlled trial (DIRECT). J Am Coll Nutr. 2009 Apr;28(2): 159-68.

26 Bradshaw AJ, Horwath CC, Katzer L, Gray A. Non-dieting group interventions for overweight and obese women: what predicts non-completion and does completion improve outcomes? Public Health Nutr. 2010 Oct; 13(10):1622-8.

27 Wadden TA, Berkowitz RI, Womble LG, Sarwer DB, Phelan S, Cato RK, et al. Randomized trial of lifestyle modification and pharmacotherapy for obesity. N Engl J Med. 2005 Nov;353(20):2111-20.

28 Fabricatore AN, Wadden TA, Moore RH, Butryn ML, Heymsfield SB, Nguyen AM. Predictors of attrition and weight loss success: results from a randomized controlled trial. Behav Res Ther. 2009 Aug;47(8):685-91.

29 Stevens J, Truesdale KP, Cai J, Ou FS, Reynolds KR, Heymsfield SB. Nationally representative equations that include resistance and reactance for the prediction of percent body fat in Americans. Int J Obes. 2017 Nov; 41(11):1669-75. 
30 Müller W, Lohman TG, Stewart AD, Maughan RJ, Meyer NL, Sardinha LB, et al. Subcutaneous fat patterning in athletes: selection of appropriate sites and standardisation of a novel ultrasound measurement technique: ad hoc working group on body composition, health and performance, under the auspices of the IOC Medical Commission. Br J Sports Med. 2016 Jan;50(1):45-54.

31 Müller W, Horn M, Fürhapter-Rieger A, Kainz P, Kröpfl JM, Ackland TR, et al. Body composition in sport: interobserver reliability of a novel ultrasound measure of subcutaneous fat tissue. Br J Sports Med. 2013 Nov; 47(16):1036-43.

32 Müller W, Horn M, Fürhapter-Rieger A, Kainz P, Kröpfl JM, Maughan RJ, et al. Body composition in sport: a comparison of a novel ultrasound imaging technique to measure subcutaneous fat tissue compared with skinfold measurement. Br J Sports Med. 2013 Nov;47(16):1028-35. 